\title{
LA INVESTIGACIÓN HISTÓRICO-EDUCATIVA EN GALICIA, 2002-2014
}

\author{
Historical-educational Research in Galicia, 2002-2014
}

\author{
Antón Costa Rico* y Xosé M. Malheiro Gutiérrez ${ }^{\S}$ \\ Fecha de recepción: 20/01/2016 • Fecha de aceptación: 21/02/2016
}

Resumen. Tomando como inicial referencia dos informes anteriores, se examina aquí el estado de la investigación histórico-educativa en Galicia y sus aportaciones desde los inicios del siglo XXI hasta los momentos actuales. Esta contribución, realizada desde los estudios y criterios generales de la sociología del conocimiento, examina la razón de dichos estudios histórico-educativos, su variable vitalidad, los problemas de investigación, temas dominantes y metodologías, los rasgos que caracterizan a sus autores, así como la presencia de tesis doctorales, monografías, capítulos de libros y artículos académicos. A la luz de los anteriores datos, los autores del examen realizan un balance interpretativo poniendo de relieve los puntos críticos que limitan esta considerable historiografía regional de la educación.

Palabras clave: Historiografía educativa. Galicia. Sociología del conocimiento. Producciones de conocimiento. Puntos críticos.

Abstract. Using two previous reports as a reference, this paper examines the status of historical-educational research in Galicia and its contributions from the beginnings of the twenty-first century to the present. This particular contribution, rooted in the general studies and criteria of the sociology of knowledge, tests the reason for the historical-educational studies mentioned, their variability, their research problems, dominant themes and methodology, together with features characterizing the authors as well as different presentations of doctoral theses, monographs, book chapters and academic articles. In light of the aforementioned data, the authors of the test carry out an interpretive balance highlighting the critical aspects that limit this considerable regional historiography of education.

Key words: Educational historiography. Galicia. Sociology of knowledge. Productions of knowledge. Critical aspects.

\footnotetext{
* Universidade de Santiago de Compostela. Facultade de Ciencias da Educación. Campus Vida. 15782 Santiago de Compostela. España. anton.costa@usc.e

${ }^{\delta}$ Departamento de Pedagoxía e Didáctica. Facultade de Ciencias da Educación. Universidade da Coruña. Campus de Elviña, s/n. 15701. A Coruña. España. xmalheiro@udc.es
}

Cómo citar este artículo: Costa Rico, Antón y Malheiro Gutiérrez, Xosé M. «La investigación histórico-educativa en Galicia, 2002-2014», Historia y Memoria de la Educación, 4 (2016): 307-335 


\section{INTRODUCIÓN GENERAL: DELIMITACIÓN Y ANTECEDENTES}

La Historia de la Educación es actualmente no solo un campo de conocimiento específico ${ }^{1}$ sino también un campo profesional en el sentido bourderiano. ${ }^{2}$ Este ejercicio investigador ha sido sometido en Galicia a un cierto y variable estudio, a modo de informe bibliométrico y con algún alcance interpretativo, en varios momentos anteriores al presente, tal como se puede observar en las referencias que se señalan, ${ }^{3}$ y se renueva mediante el presente documento, que toma por referente anterior el realizado en 2004 por Vicente Peña Saavedra, ${ }^{4}$ debiéndose anotar que determinados aspectos de la evaluación de la historiografía educativa gallega están igualmente incorporados en estudios de valoración de la historiografía educativa española, tal como se refleja en los textos que se indican al pié. ${ }^{5}$

\footnotetext{
${ }^{1}$ Que como tal puede ser examinado desde perspectivas historiográficas y desde las aportaciones de la sociología del conocimiento que, desde Karl Mannheim en particular (Essays on Sociology of Knowledge, London, 1952), abrió puertas a los estudios sobre los factores determinantes del saber, a fin de poner de relieve los lazos que subsisten, no siempre visibles, entre formas específicas de conocimiento y su difusión, por una parte, y un determinado contexto social de producción de ese conocimiento, por otra.

${ }^{2}$ Pierre Bourdieu dentro de sus estudios en relación con la episteme y con la sociología del conocimiento delineó, fuera de cualquier mirada idealista, evolucionista o ingenuamente positivista, el concepto de campo académico-profesional, como escenario contextualizado donde tiene lugar la construcción histórico-social de los saberes. Un escenario de saber-poder que permite tomar en consideración el conjunto de elementos estructurales, culturales y subjetivos que en el interior de un determinado contexto social interactúan entre si configurando un espacio social concreto.
}

${ }^{3}$ Beatriz Cebreiro López y Celestino Míguez Rodríguez (coords.), Vinte anos de investigación educativa en Galicia (1970-1990) (Santiago: Servicio de Publicacións da Universidade de Santiago de Compostela, 1992); Antón Costa Rico, «A historia da educación en Galicia. Notas para unha revisión historiográfica», Cuadernos de Estudios Gallegos, 106, (1994): 221-243; Antón Costa Rico, "La historiografía educativa en Galicia», en La investigación histórico-educativa. Tendencias actuales, eds. Antonio Viñao Frago y Narciso de Gabriel Fernández (Barcelona: Ronsel, 1997), 103-116; Antón Costa Rico, Narciso de Gabriel Fernández y Sabela Rivas Barrós, «Historiografía educativa de Galicia (17502000)», Sarmiento. Anuario Galego de Historia da Educación, 5 (2001): 147-204; Vicente Peña Saavedra, "Historia da Educación», en A investigación educativa en Galicia (1989-2001), dir. Miguel Anxo Santos Rego (Santiago de Compostela: Xunta de Galicia / Consellería de Educación e de Ordenación Universitaria, 2004) t. 2, 21-75; Xosé M. Cid Fernández, «Historiografía da educación en Ourense en 25 anos de "proximidade" ás outras disciplinas históricas», en Homenaxe a la profesora Lola F. Ferro: estudios de historia, arte e xeografía, coord. Susana Reboreda Morillo (Vigo: Universidade de Vigo, 2005.), 207-217.

${ }^{4}$ Peña Saavedra, «Historia da Educación», t. 2, 21.

${ }^{5}$ Narciso de Gabriel. «La historiografía educativa regional en España», en Repensar la historia de la educación. Nuevos desafíos, nuevas propuestas, ed. Manuel Ferraz Lorenzo (Madrid: Biblioteca Nueva, 2005), 399-422; Antón Costa Rico, «Historia de la educación en España. Realidades, problemas y tendencias en el dominio de la investigación», Cadernos de História da Educaçâo (Uberlândia, Brasil), 
Se ha apreciado en informes anteriores un incremento continuo de la producción bibliográfica histórico-educativa en sus diversas formas de presentación (libros, capítulos de libros, artículos de revistas, y textos publicados en actas congresuales) y deberíamos indicar que, en términos de volumen de unidades bibliográficas publicadas, ese incremento ha continuado durante el período 2002-2014, que aquí analizamos.

En el período 1980-2001 el total de unidades documentales censadas fue de 337. Esto es, corresponderían $16^{6}$ unidades por intervalo anual, que no ha sido así exactamente por la mayor concentración de las publicaciones en los últimos anos de la serie revisada. En el período 2002-2014 podemos registrar un acrecentado censo de 499 unidades bibliográficas; de ellas corresponderían 38 unidades por intervalo anual, con 13 años censados en la presente serie, registrándose la distribución que se refleja en el Cuadro 1 y en la Figura 1. Pero el volumen es sólo un indicio o evidencia cuantitativa que deberemos someter a valoración.

\section{Cuadro 1. Distribución de las unidades bibliográficas de Historia de la Educación}

\begin{tabular}{|c|c|c|c|c|c|}
\hline $\begin{array}{c}\text { Serie } \\
\text { cronológica }\end{array}$ & Libros & $\begin{array}{l}\text { Capítulos } \\
\text { de libros }\end{array}$ & Actas & $\begin{array}{l}\text { Artículos } \\
\text { de revista }\end{array}$ & Total \\
\hline $1980-2001$ & $67 \quad 20 \%$ & $5516 \%$ & $51 \quad 15 \%$ & $164 \quad 48 \%$ & $100 \%$ \\
\hline $2002-2014$ & $97 \quad 19 \%$ & $88 \quad 18 \%$ & $10120 \%$ & $21343 \%$ & $499 \quad 100 \%$ \\
\hline
\end{tabular}

Fuente: Elaboración propia.

10 (2), (2011): 15-44; Antón Costa Rico, «Iluminar la intensa historia de las prácticas escolares desde la historiografía educativa: potencialidad y retos», en História e Historiografía da Educaçâo Ibero-Americana: Projetos, Sujeitos e Práticas, orgzs. Claudia Alves y Ana Chrystina Mignot (Rio de Janeiro: Quartet Editora, 2012), 155-184; Antón Costa Rico, «Examinando las prácticas escolares desde la historiografía educativa», Revista de Ciencias de la Educación, 231-232 (2012): 507-516. Es deseable tener presentes dos informes de alcance español: Jean-Louis Guereña, Julio Ruiz Berrio y Alejandro Tiana Ferrer (eds.), Historia de la Educación en la España contemporánea. Diez años de investigación (Madrid: MEC/CIDE, 1994) y Jean-Louis Guereña, Julio Ruiz Berrio y Alejandro Tiana Ferrer (eds.), Nuevas miradas historiográficas sobre la educación en los siglos XIX y XX (Madrid: Ministerio de Educación, 2010).

${ }^{6}$ Procedemos al redondeo tanto en las cifras, cuanto en las distintas representaciones porcentuales presentes en este trabajo, por cuanto la precisión superior que pudieran aportar los decimales podría dificultar su lectura, sin mejorar, en cambio, la comprensión y la interpretación de los datos que se ofrecen. 
Cuál es la materia de estudio y de investigación, cuáles son los problemas examinados con más atención e insistencia en relación con la historia educativa y pedagógica por parte de los investigadores e investigadoras radicados en Galicia, qué razones y factores mueven esta historiografía, quiénes son los investigadores, a quién dirigen el conocimiento producido, qué conciencia histórica tienen de si, qué fuentes de estudio privilegian, con qué estructuras de investigación cuentan, qué resultados cualifican en mayor medida el conocimiento producido, o qué referenciales teórico-metodológicos se movilizan, habrán de ser, pues, asunto de respuesta en este informe, así como también «las formas de hacer historia», feliz expresión traducida de la obra New perspectives on historical writing de Peter Burke ${ }^{7}$ para referirse a una cierta explosión y diversificación de las formas del discurso histórico y de su comunicación, consecuencia sin duda de la diversificación de los problemas, de las fuentes utilizadas y de los métodos y técnicas usados con consciencia intelectual y rigor crítico.

\section{PRODUCCIÓN CIENTÍFICA}

\section{Artículos}

El censo de artículos publicados en revistas alcanza la cifra de 213, que obedecen a una amplia autoría: en total 71 autores y autoras (con 20 de los artículos elaborados por autorías dobles), si bien conviene señalar que sólo nueve de ellos tienen en el período una producción individual superior a las ocho unidades, reuniendo un total de 139 artículos producidos; así pues, esos autores representan solo algo más del 12\% del total, aunque reúnen casi el $65 \%$ de la producción. ${ }^{8}$ Estos nueve autores ${ }^{9}$ conforman en $^{2}$ gran medida lo que podríamos denominar «el grupo gallego de historiadores de la educación», del que forman parte algunos otros componentes

\footnotetext{
${ }^{7}$ Peter Burke, Formas de hacer historia (Madrid: Alianza Universal, 1996). Uno de los autores y textos que representan la importante renovación epistemológica en relación con lo que sea el conocimiento histórico.

${ }^{8}$ Son ellos y ellas, por el número de registros, los siguientes: Costa Rico, 28; Otero Urtaza, 21; de Gabriel Fernández, 18; Benso Calvo, 17; Peña Saavedra, 14; Malheiro Gutiérrez, 13; Porto Ucha, 11; Barreiro Rodríguez, 9, y Cid Fernández, 8.

${ }^{9}$ Sólo uno de ellos es autora, pese a que en el cómputo total de los 71 autores 26 son autoras, es decir, el 36\% del total, con el 23\% de las 210 unidades bibliográficas censadas.
} 
con una más reducida producción bibliográfica. ${ }^{10}$ Excepto en un caso más tardío, el de Malheiro Gutiérrez, ${ }^{11}$ iniciaron su actividad investigadora y de publicación a comienzos de los años ochenta, cuando hacía poco que se habían incorporado a sus labores docentes e investigadoras en los centros superiores y universitarios de Pedagogía y formación del profesorado en Galicia, o cuando daban inicio a su incorporación, y todos ellos se doctoraron en ciencias de la educación con cuidadas y eruditas investigaciones histórico-educativas defendidas a partir de 1982. Investigaciones que, de modo predominante, examinaron problemas relacionados con el pasado histórico-educativo de Galicia, así como otros ligados a la experiencia de la Segunda República y a la Institución Libre de Enseñanza. Este grupo de autores más destacados en cuanto a su producción, en cierto modo generacional, empezó a reducirse en la presente década del siglo XXI, si bien se registra una tenue aparición de algunas nuevas autorías.

Los 213 artículos se editaron en un alto y diverso número de publicaciones periódicas de muy diverso alcance y grado de reconocimiento académico, ${ }^{12}$ de carácter cultural, educativo e histórico, en setenta casos, si bien 14 de ellas (el 20\%) reúnen casi el 66\% de los artículos publicados. Una de estas revistas, Sarmiento. Anuario Galego de Historia da Educación, publicó 51 de los artículos, es decir algo más del 23\%. A distancia de esta publicación, sostenida por el que podríamos llamar «el grupo gallego», como actor colectivo, se sitúan EDUGA, ${ }^{13}$ con 15 artículos, Murguía. Revista Galega de Historia, con 13; ${ }^{14}$ Historia de la Educación. Revista Interuniversitaria, con 10; Revista Galega de Educación, con siete, y algunas

\footnotetext{
${ }^{10}$ En particular, Luis Iglesias Salvado, secretario y responsable clave de la revista Sarmiento. Anuario Galego de Historia da Educación.

${ }^{11}$ Quien presentó su tesis doctoral en 2003.

12 Algo más del 40\% de ellas indexadas en Latindex, aunque disponen de reducidos índices de impacto, de no ser los casos de Paedagogica Histórica (con contribuciones de Benso Calvo, de Gabriel Fernández y Otero Urtaza), Revista de Educación (con contribuciones de Benso Calvo y Otero Urtaza), History of Education (de Gabriel Fernández), Hispania. Revista Española de Historia (Costa Rico) y a mayor distancia Historia de la Educación (con textos de Benso Calvo, Costa Rico, de Gabriel Fernández, Otero Urtaza y Peña Saavedra).

${ }^{13}$ Publicación generalista y de difusión de educación promovida por la Consellería de Educación de la Xunta de Galicia.

${ }^{14}$ Obedece a una excepcionalidad en tanto que la publicación centrada en la historia general de Galicia dedicó monográficamente su número doble, 27/28 de 2013, a estudios relacionados con la historia de la educación en Galicia, como un reconocimiento a esta dimensión de la investigación impulsada sobre todo desde el campo pedagógico.
} 
otras, entre las que debemos señalar Paedagogica Histórica, de carácter internacional, con cuatro, y la Revista de Educación, con otros cuatro artículos. Las publicaciones académicas especializadas en los ámbitos pedagógico e histórico representan más de la mitad de las 70 cabeceras referidas, al lado de las otras publicaciones generalistas, ${ }^{15}$ culturales,${ }^{16} \mathrm{de}$ historia eclesiástica ${ }^{17} \mathrm{y}$ de historia local. ${ }^{18} \mathrm{Y}$ hay que señalar que fuera de los casos de las publicaciones Sarmiento. Anuario Galego de Historia da Educación, Historia de la Educación y Paedagogica Histórica, académicas y especializadas en la historia de la educación, que reúnen 66 de los artículos, esto es, el 30\% de lo publicado, podemos registrar una presencia no menor de artículos publicados en una amplia nómina de revistas gallegas ${ }^{19}$, españolas ${ }^{20} \mathrm{o}$ de otros países, ${ }^{21}$ también académicas y del ámbito educativo o del histórico. Algo más de la mitad son revistas editadas en Galicia, siendo 11 las editadas fuera del Estado español. Algo más del $40 \%$ de las 70 cabeceras $(\mathrm{N}=29)$ son revistas de educación o singularizadas en la historia educativa.

En cuanto a la lengua de los textos es necesario anotar que 117 artículos han sido editados en gallego, ${ }^{22}$ esto es, el 55\% del total, al lado

\footnotetext{
${ }^{15}$ Por ejemplo: las gallegas Revista Galega de Educación y EDUGA, o Innovación Educativa.

16 Por ejemplo: Grial o Boletín Galego de Literatura.

17 Por ejemplo: Hispania Sacra, Auriensia, Lucensia, Compostellanum o Estudios Mindonienses.

18 Por ejemplo: el Anuario Brigantino, Cátedra, Raigame, Revista de Estudos Miñoranos, Glaucopis, A Estrada, Boletín Auriense, Pontevedra. Revista de Estudios Provinciais, Argentarium o Descubrindo Deza.

19 Por ejemplo: Minius, Revista Obradoiro de Historia Moderna, Revista Galego-Portuguesa de Psicoloxía e Educación, Estudos Migratorios, o Cuadernos de Estudios Gallegos, todas ellas de carácter universitario.
}

${ }^{20}$ Por ejemplo: Revista Española de Filosofía Medieval, Revista de Innovación en Educación, Revista de Educación, Estudios de Educación, Revista de Ciencias de la Educación, Revista Española de Pedagogía, Cuestiones Pedagógicas, Bordón, la reciente Historia y Memoria de la Educación, Educació i História, Boletín de la Institución Libre de Enseñanza, o Hispania. Revista Española de Historia.

${ }^{21}$ Ricerche Pedagogiche e History of Education \& Children's Literature, de Italia; Solska Kronica, de Polonia; Historia de Portugal; Memoria, Conocimiento y Utopía e Historia de la Educación Argentina, de Argentina, y Educaçâo e Filosofia, Cadernos de História da Educaçâo, Revista Diálogo Educacional, Pro-posiçôes, y Revista Brasileira de Historia da Educaçâo, de Brasil, totalizando en este caso once contribuciones elaboradas, en orden decreciente, por Peña Saavedra, Costa Rico y de Gabriel Fernández.

22 Publicados en medida muy notable en Sarmiento. Anuario Galego de Historia da Educación, aunque también presentes en otras publicaciones gallegas, o en algún caso en Brasil. Todos los miembros del "grupo gallego» desarrollaron publicística en gallego, más declaradamente en algunos casos. 
del $37 \%$ realizado en castellano, pero solo el $2.80 \%$ lo fué en inglés, lo que es indicio de la escasísima «internacionalización» de la investigación histórico-educativa realizada en Galicia.

Es oportuno indicar el amplio predominio de la autoría única de los artículos, algo que, por otra parte, resulta muy visible si nos referimos a ese grupo de nueve autores con una presencia sostenida en cuanto a la publicación de textos. Se trata, por otra parte, de profesorado universitario en más amplia medida, y del ámbito pedagógico más que del histórico general, aunque hay un porcentaje de autores y autoras que son docentes de educación secundaria con distinta especialización en los estudios históricos.

Los objetos de estudio examinados tienen casi en un 70\% como referencia central a Galicia, en su conjunto, o a alguno de sus territorios locales, comarcales o provinciales, pero en algo más de un $30 \%$ estudian, analizan o exponen aspectos con referencia española o también internacional. Hay que decir que con alguna frecuencia no se eluden las limitaciones de lo que debe ser entendido como una historiografía "localista», como tal limitada, pero hay que destacar el predominio de una historiografía que evita el localismo, por más que sus objetos de estudio tengan como referencia central temáticas centradas en Galicia.

Menos favorable puede resultar la valoración del encuadre temporal de los objetos de estudio de los 213 artículos tomados en consideración. Un $60 \%$ de los trabajos focalizan su temática exclusivamente en el siglo xx; un $16 \%$ lo hace en el siglo XIX; un 11.6\% en el tiempo del Antiguo Régimen, con mayor querencia para el tiempo del siglo XVIII y la general temática de la Ilustración, más en particular; un 12\% aborda temáticas que aprehenden en su conjunto los siglos XIX y XX, casi siempre por atender a asuntos del tiempo de entre siglos, y del previo a la Segunda República española. Sólo un artículo, en cada caso, tiene que ver con períodos anteriores (Antigüedad, Edad Media, Renacimiento).

Hablemos de la temáticas de estas distintas y desiguales contribuciones. ${ }^{23}$ Los trabajos referidos al profesorado - con mayor atención

\footnotetext{
${ }^{23}$ No es fácil establecer una categorización de las temáticas posibles de los trabajos siguiendo un esquema de clasificación global, al tiempo que suficientemente analítico y con frecuencia no es fácil encajar cada uno de los trabajos examinados en una específica categoría. Cuando esto no sucede con nitidez se adopta el criterio de asignar un dato a aquella categoría a la que en mayor grado responde
} 
al tiempo del primer tercio del siglo xx y Segunda República (por este orden: reconstrucciones biográficas específicas, formación inicial, profesionalización, renovación pedagógica, societarismo/sindicalismo, y sociología histórica)—, en número de 39, alcanzan el 18\% de la producción académica. Un $17 \%$ de los trabajos, en número de 37 , tienen que ver con la historia de la pedagogía (corrientes, prácticas y figuras transcendentes, como podrían ser Rousseau, Luzuriaga, Cossío, Giner, o también el Padre Sarmiento o María Barbeito, entre otras). La atención a la problemática de la alfabetización, distintos aspectos referidos a la educación popular y en particular la cuestión de las escuelas suscitadas por los emigrantes, está presente en 28 contribuciones. Siguen, con 16 aportaciones, los trabajos relacionados con la historia del currículum (materias, contenidos, áreas; recursos, manuales y materiales didácticos; metodología e innovaciones didácticas), a las que se podrían sumar las 14 relacionadas con el patrimonio escolar, a partir de los trabajos realizados desde el Museo Pedagógico de Galicia. Dieciseis de las contribuciones podrían agruparse como "historia institucional», en tanto que atienden particularmente a examinar distintas instituciones de distinto nivel académico. Trece de los trabajos tienen como objeto distintos aspectos sociales, ideológicos y relativos a las políticas educativas y sus programas.

La problemática y realizaciones referidas a la educación de la mujer están presentes en 11 de las contribuciones: la cuestión del género, de los roles o de la feminización y formación del profesorado femenino goza de alguna presencia. La cuestión de la identidad cultural y de la lengua gallega es central en 10 trabajos y en otros tantos la educación secundaria o también la formación en seminarios, a todo lo que podríamos añadir las cinco contribuciones relacionadas con la docencia y la historiografía educativa, las cinco referidas a la historia de la literatura infantil, las cuatro sobre el estatuto de la infancia y algunas otras.

El nivel de la educación primaria, el más extendido sociológicamente, es el más presente, seguido a mucha distancia por trabajos centrados en la educación secundaria (con preferencia nítida para los institutos de bachillerato), mientras que apenas hay textos relativos a la educación

su contenido, siendo conscientes de una cierta aleatoriedad en la operación. Por eso, tanto el número de artículos de cada agrupación de asuntos, como su peso porcentual relativo en el total, debe ser considerado como indicativo y orientativo, desde la búsqueda de la mayor objetividad. 
infantil, universitaria, técnico-profesional, especial, especializada, artística o de idiomas.

Para una mayor transparencia en cuanto a las temáticas estudiadas en estos artículos haremos una observación analítico-sintética en relación con las aportaciones de los autores con mayor producción individual, a la que añadiremos algunas notas complementarias. Barreiro Rodríguez centró sus trabajos en las figuras de Rousseau y su relación con los inicios de la educación pública, y de Luzuriaga, el gran impulsor de las políticas de reforma educativa republicana. ${ }^{24}$ Benso Calvo atendió de forma destacada a la educación secundaria, a su profesorado y libros de texto, al currículum de la materia de urbanidad, a la práctica docente en el tiempo del franquismo y a la coeducación y educación de las mujeres en Ourense. Costa Rico examinó la historiografía educativa, las figuras del Padre Sarmiento (en colaboración con Álvarez Lires) de X. V. Viqueira y de Ramón de la Sagra, la cuestión de la identidad cultural y lingüística en Galicia, la represión del profesorado republicano por el franquismo, la formación del profesorado y la renovación pedagógica entre el franquismo y la transición democrática. Narciso de Gabriel analizó desde diversos ángulos la conexión entre la alfabetización y la escolarización, así como la conexión entre la cultura social popular y la cultura escolar, prestando también atención a la profesionalización del magisterio y a las figuras de Álvarez Blázquez y Castelao. Xosé M. Malheiro atendió el asunto de la sociabilidad, la política, la cultura y la educación entre los emigrantes gallegos a América y su acción en Galicia, con atención a las figuras de Ares de Parga y de Alonso Ríos. Fueron examinadas por Otero Urtaza las relaciones de Giner y de Cossío, desde la Institución Libre de Enseñanza (ILE), con la intelectualidad europea y americana, las Misiones Pedagógicas, así como las figuras de Luis Hermida y Ramón Varela. Peña Saavedra, como coordinador científico que fue del Museo Pedagógico de Galicia, escribió distintos textos sobre los museos y el patrimonio escolar (memoria, saberes y cultura escolar), con nuevas aproximaciones a la acción escolar de los emigrantes gallegos. Porto Ucha, que elaboró nuevas contribuciones sobre la ILE en sus relaciones con Galicia, abordó en varios momentos la formación y la profesionalización del magisterio

${ }^{24}$ El profesor Herminio Barreiro Rodríguez, que fue durante varias décadas profesor de la materia de Historia da Educación en la Universidad de Santiago (desde el curso 1977-78 hasta el 2003-04) falleció en noviembre de 2010. 
(en colaboración con Iglesias Salvado) y la represión política franquista sobre los maestros pontevedreses. Esta última cuestión con referencia a la provincia de Ourense y otros aspectos sobre su profesorado en el tiempo de la Segunda República estuvo en el centro de las aportaciones de Cid Fernández.

En el período considerado aparecieron diversas contribuciones sobre: el pasado escolar de lugares como A Estrada, Padrón, Ourense o Celanova, entre otros; la represión franquista sobre el profesorado; la interrelación entre oralidad y cultura escrita; las Escuelas Normales de Galicia; biografías de maestros republicanos; la cultura letrada, la alfabetización y las escuelas en la Galicia moderna; la utilización de los expedientes del alumnado como fuente para la historia de la educación; la formación eclesiástica; la formación monástica benedictina; el papel de la literatura infantil gallega en relación con la galleguización escolar, y la educación de niños ciegos y de hospicianos, entre otras.

\section{Libros}

En la serie temporal 2002-2014 se editaron 97 libros relativos a la historia de la educación a cargo de 62 investigadores y autores de Galicia (de ellos el 29\% mujeres), versando una parte muy notable sobre aspectos de la historia educativa de Galicia. Algo más del $80 \%$ son de autoría única. Cuatro de los textos son ediciones introducidas. Diez de los 97 títulos son obras de carácter colectivo, que cuentan con uno o varios directores, coordinadores o compiladores. Más allá de la dispersión de las autorías, hay varios autores que reúnen tres o más textos en el período: Malheiro Gutiérrez, siete; Peña Saavedra, cinco; Costa Rico, cinco; y tres, Benso Calvo, Cid Fernández, Franco-Grande, Gurriarán Rodríguez, Porto Ucha y Varela Orol; esto es, nueve autores (el 15\%) alcanzan el 38\% de la producción, de un modo similar a lo sucedido en el caso de los artículos de revistas. El $86 \%$ de los textos publicados $(\mathrm{N}=79)$ se editaron en Galicia, sobre todo a cargo de editoras institucionales, ${ }^{25}$ más que de editoras privadas.

En algo más de dos tercios, se trata de libros de carácter monográfico en cuanto a su temática, frente a aquellos otros que podríamos agrupar

\footnotetext{
${ }^{25}$ Universidades y Diputaciones, en particular, más que la propia Xunta de Galicia, con alguna mayor preferencia de la Universidad de Santiago, con once referencias.
} 
bajo el rótulo de «estudios generales». En un 59\% deben ser considerados como el resultado de estudios de investigación, frente a los que podríamos definir de «difusión». El 42\% de ellos tienen al conjunto de Galicia como sujeto de estudio en el ámbito histórico educativo; poco más de un $10 \%$ se circunscribe a un determinado espacio provincial, siendo más numerosos aquellos que podríamos encuadrar como estudios locales en Galicia; un 10\% de los 97 títulos se refieren a problemáticas educativas de alcance general español, y un 5\% de ellos tienen un carácter transnacional. Casi un $60 \%$ están escritos y editados en lengua gallega, frente a un $30 \%$ en castellano.

Sus temáticas y problemas de estudio en ningún caso se refieren a la Baja Antigüedad ni al conjunto de la Edad Media, ni al tiempo del Humanismo renacentista, con la excepción de un caso, ${ }^{26}$ considerado con alguna excepcionalidad por el tipo de obra. Siendo así, los libros se centran en particular en el siglo xx (desde su inicio) con el 58\% de los títulos; un 14\% de ellos atienden al siglo XIX y un 13\% al amplio período del Antiguo Régimen, con más atención al siglo XVIII que a momentos anteriores.

Los asuntos abordados son variados, como no podía ser de otro modo, aunque podríamos singularizar una serie de cuestiones, distintamente abordadas: la problemática de la emigración a América, sobre todo durante el siglo XIX y el primer tercio del siglo XX en relación con la sociedad gallega, la alfabetización, la educación popular, el asociacionismo y la escolarización es el asunto de 13 de los libros; el estudio del profesorado primario, en particular, abordado en relación a su estatuto, formación y testimonios biográficos se advierte en 16 libros; la preocupación por el desarrollo científico en Galicia en relación con la Universidad, con la Ilustración y con los avances decimonónicos es abordada por once libros. Otros asuntos atraen, en cada caso, el siguiente número de libros: siete, la cuestión mujer y la educación; seis, los problemas de política educativa, en particular, la alfabetización y la escolarización; seis, el pensamiento pedagógico; seis, las cuestiones relacionadas con el patrimonio material escolar; y siete, la historia de diversas instituciones educativas de educación primaria y secundaria. Centran la atención de cinco libros

\footnotetext{
${ }^{26}$ Antón Costa Rico, Historia da educación e da cultura en Galicia (séculos IV-XX). Permanencias e cambios no contexto cultural e educativo europeo (Vigo: Edicións Xerais de Galicia, 2004).
} 
las cuestiones relacionadas con la lectura, los libros y la cultura escrita. Tres ediciones en cada caso atienden a asuntos como las dinámicas académicas y estudiantiles en la Universidad de Santiago durante el siglo XX (espacio de investigación privilegiado por las contribuciones de Ricardo Gurriarán), algún segmento de la formación profesional, la llamada cultura popular y las ediciones introducidas. Otros seis textos atienden a variados asuntos.

A estos efectos, se han movilizado numerosas y distintas fuentes documentales primarias con empleo de los recursos y fondos existentes en los diversos archivos y centros documentales de Galicia, que enunciamos sin otra especificación: documentos manuscritos, prensa general y especializada, documentación institucional y administrativa, memorias de vida, registros académicos universitarios, series estadísticas españolas, archivos gráficos, registros notariales, fondos bibliotecarios específicos (como el archivo jesuíta de Galicia), patrimonio escolar, y distinto tratamiento de textos, acudiendo para eso no sólo a los más reconocidos fondos de archivo existentes en Galicia, sino también a espacios como la Biblioteca Nacional, el Archivo Nacional, archivos franceses, la Real Academia de la Historia, la Residencia de Estudiantes, o diversos archivos locales, debiendo también mencionar el creciente auxilio ofrecido por bases de datos digitalizadas y presentes en la Red.

La visible diversificación de las fuentes primarias nos ofrece indicios sobre la relativa riqueza metodológica histórica desde la que se elaboraron una parte, por lo menos, de estos 97 textos, como consecuencia de la toma en consideración de los debates epistemológicos suscitados tan vivamente en las últimas décadas del siglo xx en el campo de la investigación histórica. Así, podríamos afirmar que la historiografía educativa realizada en Galicia en estos años iniciales del siglo XXI está totalmente distanciada tanto del idealismo histórico, como del historicismo. Está elaborada, generalmente, desde los criterios de un positivismo historiográfico matizado y en alguna medida desde el estructural-funcionalismo, bajo una cierta influencia de los modos de hacer historia derivados genéricamente de la Escuela francesa de los Annales. ${ }^{27}$ Una historiografía

${ }^{27}$ Recordando «la nouvelle Histoire», que se abrió paso con la trilogía de textos que en 1974 impulsó Pierre Nora (Faire de l'histoire). 
de acento social, con eclecticismo teórico-metodológico, ${ }^{28}$ distanciada del materialismo histórico, pero con algún contagio, un algo impreciso, de la historia social británica con su visión dialéctica de las relaciones entre infraestructura y superestructura, sometida a alguna influencia pos-estructuralista. Una historiografía que ofrece algunos modos de hacer próximos a la nueva historia cultural, practicada a partir de la ultima tournant de la Escuela de los Annales.

Son ejemplos de este vario y más rico modo de historiar obras como las siguientes, también indicadas en el Cuadro 2: la de María Álvarez Lires, Sarmiento: un científico de la segunda Ilustración (2002), elaborada desde la toma en consideración de los supuestos de la «historia social de la ciencia»; la de Carmen Benso Calvo, Profesores y textos en el bachillerato. Uso y producción de obras de texto en los institutos gallegos del siglo XIX (2003), que busca examinar una fuente histórica tanto tiempo desconsiderada, los libros de texto, guía próxima y exponente de las prácticas

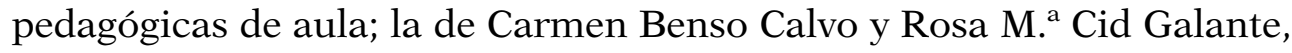
Entre a tradición e o cambio en Galicia. As mulleres na sociedade ourensá do comezo do século XX (2011) y la de Raquel Vázquez Ramil, Mujeres y educación en la España Contemporánea: la Institución Libre de Enseñanza y la Residencia de Señoritas de Madrid (2012) que se enfrentaron a otra cuestión tan relevante como silenciada en relación con la educación femenina y el género; la de Antón Costa Rico, Historia da educación e da cultura en Galicia. (séculos IV-XX). Permanencias e cambios no contexto cultural e educativo europeo (2004), obra de investigación, pero también de síntesis global, que es una narración de la historia cultural-educativa de Galicia como sujeto histórico, construida desde la asunción de los supuestos historiográficos de la nueva historia social; la de Isidro Dubert, Cultura popular e imaxinario social en Galicia (2007), solvente expresión de la incorporación, entre nosotros, de los estudios y categorías de Bajtin y del debate sobre la cultura popular en interrelación con los discursos del poder; la de Narciso de Gabriel Fernández, Ler e escribir en Galicia. A alfabetización dos galegos e das galegas nos séculos XIX e XX (2006), obra

\footnotetext{
28 Los autores queremos ofrecer aquí un punto de vista más matizado que el más genérico de «análisis cuantitativo o análisis cualitativo», que con frecuencia se utiliza para contraponer los abordajes de tipo positivista a los de tipo hermenéutico, como si de una rígida dicotomía se tratase. El enfoque cuantitativo y el cualitativo evidencian, en todo caso, dimensiones distintas («explicar»/«comprender»), que podrían ser complementarias y de facto aparecen con frecuencia en una misma investigación, de no mediar enfoques paradigmáticos/teóricos distintos.
} 
que guarda conexiones con el texto de Dubert y que se sumerge en la problemática histórica de la alfabetización, una de las que con Harvey Graff, Furet/Ozouf o Carlo Cipolla marcó la renovación de la historiografía educativa occidental.

Igualmente, hay que mencionar las obras de Narciso de Gabriel, Elisa e Marcela. Alén dos homes (2008), que significa la recuperación crítica de la biografía como valiosa narración histórica, modalidad esta también cultivada por Anxo. S. Porto Ucha, en Historias de vida. $O$ maxisterio pontevedrés na II República, Guerra Civil e comezos do Franquismo (2003) y por Ana Romero Masiá, en María Barbeito. Unha vida ao servizo da escola e dos escolares (1880-1970) (2014); la de Ricardo Gurriarán, Ciencia e conciencia na Universidade de Santiago (1900-1940) (2006), quien anuda historia institucional, historia social e historia de la ciencia, bien lejos de las tradicionales y positivistas historias institucionales universitarias; la de Xosé M. Malheiro Gutiérrez, As escolas de emigrantes e o pensamento pedagóxico: Ignacio Ares de Parga e Antón Alonso Ríos (2006), quien indaga en los discursos y en las ideologías para observar la complejidad de un aparente fenómeno histórico unitario; la dirigida por Vicente Peña Saavedra, Los museos de la educación en Internet (2004), que nos muestra la importancia del uso de las tecnologías de la información al servicio de la investigación histórica; las de Ofelia Rey Castelao, Libros y lectura en Galicia. Siglos XVI-XIX (2003), y de Concepción Varela Orol y Martín González Fernández, Heterodoxos e Malditos. Lecturas prohibidas na Universidade de Santiago (2002), que re-visitan desde la nueva historia social cultural lo que en otro tiempo era examinado con criterios emanados desde el idealismo histórico y el racionalismo filosófico; la de Ana Sánchez Bello, $O$ traballo das mestras en Galicia: entre a produción e a reprodución (2006), que realiza desde Galicia un infrecuente ejercicio de sociología histórica; la de Mercedes Suárez Pazos, Xosé M. Cid y Carmen Benso, Escola e memoria (2006), quienes se aproximan a los métodos etnográficos y fenomenológicos con intención de ahondar con mejores armas interpretativas la realidad escolar, la vida de las aulas; o la de Manuel F. Vieites, Creación dramática, educación popular e construción nacional (2006), quien ha podido incorporar la de-construcción derridiana y los estudios literarios históricos a la hora de examinar desde nuevas perspectivas el ámbito de la educación popular. 
En síntesis, todo un conjunto de expresiones del hacer histórico que forma parte de la agenda de problemas, interrogantes y modos actuales de historiar en el contexto occidental.

\section{Capítulos de libros}

En la serie temporal 2002-2014 la historiografía educativa elaborada desde Galicia tiene una fuerte manifestación y presencia en el contexto de los capítulos de libros de autoría colectiva al alcanzar un total de 185 trabajos editados (el 38\% de la producción total de textos), escritos por parte de un número más restringido de autores y autoras que en el caso de la edición de libros de autor único/doble o en el de la publicación de artículos: en total 39, confirmándose de nuevo la existencia de un «grupo gallego de Historia de la Educación", al considerar la concentración de los trabajos elaborados en un reducido y coincidente grupo de autores. En algo más del 90\% hablamos de trabajos de autor único, pues solo 16 de ellos han sido publicados por dos autores y solo uno por tres autores. Del total, trece son mujeres, es decir, el 34\%.

Es necesario señalar que más del 50\% de estos textos $(\mathrm{N}=101)$ se han publicado en libros de actas de reuniones congresuales directamente referidas a la Historia de la Educación, de alcance estatal o internacional (en un reducido número de casos), celebradas a lo largo de esta serie temporal fuera de Galicia. ${ }^{29}$ Que esto sea así pone de relieve que la historiografía construida desde Galicia está interconectada a través de la participación de los historiadores de Galicia en estos encuentros de intercambio académico.

En cuanto a la temporalidad alcanzada por los problemas estudiados, es necesario resaltar de nuevo la intensidad de asuntos situados en el siglo Xx (con 141 trabajos, o dicho de otro modo, el 77\% del total). Menos

\footnotetext{
${ }^{29}$ Con la excepción de la celebración en Santiago de las I Jornadas Científicas de la Sociedad Española para el Estudio del Patrimonio Histórico-Educativo (SEPHE) y de las IV Jornadas de Institutos Históricos, ambas celebradas en el Museo Pedagógico de Galicia respectivamente en 2005 y en 2010. Con anterioridad ya se habían celebrado en Galicia el VI Coloquio Español de Historia de la Educación (1990, Santiago de Compostela) en colaboración con la Sociedad Española de Historia de la Educación (SEDHE), el IV Encuentro Ibérico de Historia de la Educación (2001, Allaríz) y el I Foro Ibérico de Museísmo Pedagóxico. O museísmo pedagóxico en España e Portugal (2001, Santiago de Compostela, MUPEGA).
} 
de un $4 \%$ se refieren a un tiempo anterior al siglo XIX. Habla esto de la construcción de una Historia de la Educación muy altamente ligada al desarrollo del sistema educativo español.

En cuanto al espacio acogido por los distintos trabajos, es preciso anotar que un 30\% de ellos se refieren a Galicia como conjunto, siendo casi un $30 \%$ los que lo hacen al contexto español. Un 12\% de los trabajos se restringe a una de las cuatro geografías provinciales de Galicia, acercándose al 16\% aquellos que se refieren a espacios delimitados localmente (ciudad, villa, comarca), mientras un $7 \%$ muestra un alcance internacional.

Podemos anotar, como dato de interés, el predominio de los trabajos que aportan conocimiento nuevo, como fruto de investigación, sobre los que podríamos considerar trabajos de difusión, descendiendo aquí el número de los publicados en lengua gallega ( $\mathrm{N}=80$, esto es, el $43 \%)$, al tiempo de registrarse un aumento en el número de textos editados en castellano, como consecuencia, en particular, de que muchos de tales textos revisten la forma de comunicaciones presentadas en los encuentros académicos de Historia de la Educación celebrados en el período. Sólo siete del total de los textos han sido publicados en otras lenguas: francés (tres), inglés, italiano, portugués y catalán (uno en cada caso).

Hablemos de las temáticas de estas 185 contribuciones. Los trabajos referidos a distintos aspectos sociales, ideológicos y relativos a las políticas educativas suman un total de 35 (un 19\%), destacando en particular los que se refieren a la política educativa y a su administración. Son 27 las contribuciones que podríamos encuadrar estrictamente como estudios de historia pedagógica (corrientes, prácticas, figuras transcendentes). Un número un poco inferior ( 25 trabajos, un 13\%) están relacionados con el profesorado, de nuevo con mayor atención al tiempo del primer tercio del siglo xx y la Segunda República (por este orden: reconstrucciones biográficas específicas, formación inicial, profesionalización, renovación pedagógica, societarismo/sindicalismo, sociología histórica y prensa pedagógica). Veinte de las contribuciones (un 11\%) están referidas a historia del currículo (materias, contenidos, áreas; recursos, manuales y materiales didácticos; metodología e innovaciones didácticas) sin que haya en esto alguna singular particularidad, de no ser las varias aportaciones relacionadas con el patrimonio escolar a partir de los trabajos realizados 
por el Museo Pedagógico de Galicia. Dieciocho trabajos tienen que ver con el fenómeno de la emigración a América, la educación popular y los colegios llamados «de los americanos». Otros catorce podrían agruparse como "historia institucional», en tanto que atienden particularmente a examinar distintas instituciones (escuelas, institutos, seminarios, o colegios privados religiosos en particular).

El nivel de la educación primaria es el más observable en los trabajos, con muy reducida presencia de trabajos centrados en la educación secundaria, la educación infantil, la universitaria y la educación especial (solo dos textos), y con silencio en lo que se refiere a otras modalidades de formación. Es necesario señalar en este ámbito, la escasa producción de textos en relación con las mujeres y la educación (solo cuatro) si nos referimos a las prácticas escolares.

Siete de los 185 trabajos están relacionados con la docencia de la Historia de la Educación, con el análisis de fuentes, con la metodología histórico-educativa o con revisiones historiográficas. Sólo cinco de las aportaciones abordan la cuestión de la identidad cultural y lingüística y la problemática escolar-educativa que en Galicia esto representa. Por fin, anotamos cuatro aportaciones acerca del estatuto y representación de la infancia de los niños gallegos. Dos relacionadas con la arquitectura escolar y otras dos con aspectos de la formación extraescolar. Asuntos como la política educativa republicana, las Misiones Pedagógicas, la ILE o la represión política franquista del profesorado fueron examinados con atención, entre algunos otros como las escuelas de los emigrantes o el ya citado patrimonio escolar.

Entre los 39 autores y autoras hay nueve que reúnen un total de 154 textos (16 de ellos en colaboración), esto es, el 84\% del total de textos escritos: Benso Calvo, Cid Fernández, Costa Rico, de Gabriel Fernández, Malheiro Gutiérrez, Otero Urtaza, Peña Saavedra, Porto Ucha y Vázquez Ramil, listado este que con leves modificaciones replica el señalado con anterioridad. Autores éstos con algunas temáticas predominantes; en el caso de Benso: textos escolares de urbanidad, el desarrollo de la educación secundaria, la metodología docente y las mujeres y la educación; en el de Cid hay una nítida predilección por el estudio del tiempo de la Segunda República y el profesorado «republicano» ourensano; en el de Costa hay una mayor amplitud de cuestiones: las figuras del Padre 
Sarmiento y La Sagra, la renovación pedagógica y el profesorado tanto durante el primer tercio como en el final del franquismo y en el último tercio del siglo xx, la cuestión de la lengua gallega y de la identidad cultural, la historia del pensamiento pedagógico, y la historiografía educativa; en el de Gabriel destacan nuevamente sus aportaciones sobre la alfabetización en Galicia, con alguna atención a la historiografía y a las escuelas de los emigrantes; en el de Malheiro es esta última cuestión, la de las escuelas de los emigrantes, a la que dedicó mayor atención, junto con las historias de vida del profesorado; en el caso de Otero hay que destacar sus aportaciones en relación con la ILE, la política educativa republicana y las Misiones Pedagógicas, junto a una creciente atención a la educación no formal; en el de Peña destacan tres amplias cuestiones: escuelas y cultura de los emigrantes, museismo escolar y pedagógico y periodismo infantil; Porto Ucha y Vázquez Ramil han atendido al desarrollo de la ILE, a las mujeres gallegas presentes en la Escuela Superior del Magisterio en Madrid, al profesorado en el tiempo de la Segunda República y a la represión franquista, con alguna atención al desarrollo local pontevedrés de la educación.

Más allá de esta agregación de asuntos podemos encontrar piezas singulares: desde la formación de los escribanos en el Antiguo Régimen, hasta la constitución de un archivo de imágenes significativas para la docencia de la historia de la educación (Bolaño Amigo), pasando por textos sobre el mundo de la infancia popular en Galicia, los maestros laicos, algún examen de cuadernos escolares, la representación de los niños en Castelao, la conservación del patrimonio escolar, las influencias de la psicología educativa internacional en los comienzos del siglo xx, algunas ediciones de textos, la explotación de las memorias de oposición docente, o el ajuar de la escuela. Todo un universo de información y de conocimiento, que puede ser base para estudios revisados, ampliaciones y pista para nuevas investigaciones.

\section{Tesis doctorales}

En este tiempo fueron nueve las tesis doctorales presentadas y dirigidas como trabajos de investigación desde las tres Universidades gallegas, correspondiendo siete de ellas a la Universidad de Santiago (cinco dirigidas por Costa Rico) y una a cada una de las otras univer- 
sidades. Siete de las tesis presentan un problema de estudio relativo a Galicia y dos a Portugal. La educación de la mujer, las escuelas de los emigrantes gallegos y el pensamiento pedagógico, la educación de menores en riesgo, los colegios privados femeninos, la escolarización en la ciudad de Vigo, así como aspectos de la historia del currículum de física y química y de la educación física, fueron los motivos de las siete anteriores tesis.

\section{DINÁMICAS DEL CAMPO}

\section{Proyectos, redes y contratos de investigación}

Es necesario señalar que en este período examinado no se registra la existencia de ningún proyecto de investigación histórico-educativa realizado bajo contrato económico público. Entre las razones de ello cabe destacar: una cierta dificultad de los investigadores distribuidos entre las tres universidades de Galicia, para conformar un núcleo investigador suficientemente agregativo desde el punto de vista temático; la considerable y distinta especialización que en cada caso han ido logrando en relación con cuestiones que requieren alta formación, al punto de caracterizar con alguna singularidad el oficio intelectual de historiador; ${ }^{30} \mathrm{o}$ la reducción registrada en las titulaciones universitarias en cuanto a los contenidos formativos de carácter histórico-educativo bajo la influencia de corrientes presentistas y utilitaristas, que, del mismo modo, condicionan la limitada conciencia administrativa pública acerca del valor de la investigación histórica.

Sin embargo, es evidente la existencia y visibilidad de la investigación histórico-educativa en Galicia. La publicación Sarmiento. Anuario Galego de Historia da Educación es testimonio, para este tiempo que examina-

\footnotetext{
${ }^{30}$ El oficio intelectual de historiar, situado en el ámbito de las ciencias sociales, en cuanto pide notable formación panorámica, además de especializada, a fin de explicar, comprender e interpretar aquel o aquellos fenómenos históricos examinados, siendo conscientes, por una parte, de la diversidad paradigmática y de la riqueza conceptual y teórica (una compleja caja de herramientas), y por otra, del específico punto de vista asumido por el historiador en el examen investigador a realizar, dificulta la constitución de equipos de investigación, dado que sus integrantes deben estar suficientemente compactados intelectualmente. En parte debido a todo esto, sobresale en la tradición historiográfica occidental la notable presencia de autorías singulares en cuanto a proyectos y presentaciones de la investigación, por encima de la existencia de proyectos colectivos de investigación que, sin embargo, son deseables y, sin duda, también existen.
} 
mos, de una efectiva red de investigación, aún contando con su reducida formalización.

Es oportuno, en todo caso, resaltar la existencia del Museo Pedagóxico de Galicia. Un proyecto impulsado desde el año 2000 por la Consellería de Educación de la Xunta de Galicia, bajo la coordinación científica del profesor Vicente Peña Saavedra (2000-2005). El MUPEGA abrió sus puertas al público en 2004 con un detallado programa expositivo y con varios proyectos de investigación referidos a la recuperación, conservación, análisis y difusión del patrimonio escolar de Galicia, poniéndolo de relieve mediante distintas publicaciones y ediciones digitales. Este proyecto ha reducido, más tarde, sus primeros impulsos.

\section{JORNADAS CIENTÍFICAS Y OTRAS INICIATIVAS}

Debemos destacar aquí la serie continua de Xornadas de Historia da Educación celebradas en Foz, bajo la dirección del profesor Eugenio Otero Urtaza, el Congreso Emigración e Educación celebrado en Gondomar (Pontevedra) en 2009, bajo la coordinación del profesor Xosé M. Malheiro, la celebración de las Primeras Jornadas científicas para el Estudio do Patrimonio histórico-educativo celebradas en Santiago de Compostela en 2005, el Primeiro Encontro Iberoamericano de Museos Pedagóxicos e de Museólogos da Educación, igualmente celebrado en Santiago en 2008, bajo la coordinación del profesor Vicente Peña y, finalmente, la serie de Congresos Manuel L. Acuña, celebrados en Pobra de Trives, que en sus cinco convocatorias, siendo uno de sus coordinadores el profesor Xosé M. Cid, manifestó una atención continuada a aspectos de la historia de la educación en Galicia. Cabría también destacar, dada su excepcionalidad, la edición en tres volúmenes dirigida por el profesor Xesús Rodríguez Jares, Educación e Paz. 25 anos de educación pola paz, (Vigo: Xerais, 2008) que deja constancia de las intensas dinámicas realizadas en el campo de la educación para la paz desde 1983 en Galicia.

En cuanto a otras iniciativas, podemos señalar que Xosé M. Cid ha participado en la realización de varios homenajes públicos a distintos profesores y profesoras, acompañando diversas iniciativas de recuperación de la Memoria Histórica en relación con el republicanismo de la Segunda República y la represión franquista del profesorado en la pro- 
vincia de Ourense. En este tipo de iniciativas también se implicaron en distintos puntos de Galicia, en particular, los profesores Herminio Barreiro Rodríguez, Ana Romero Masiá, Ricardo Gurriarán (en este caso, con respecto a la represión política de profesores de la Universidad de Santiago), Ángel Porto Ucha y Antón Costa Rico. Al profesorado republicano se le rindió homenaje colectivo en las instalaciones del Museo Pedagóxico de Galicia el 21 de junio de 2006.

Antón Costa Rico, como Presidente de la comisión organizadora del IV Encuentro Ibérico de Historia de la Educación (con los profesores Carmen Benso y Narciso de Gabriel), celebrado en Allariz (Ourense) en septiembre de 2001, coordinó, junto con José Luis Iglesias Salvado, la edición de sus Actas: «La mirada del otro. Para una historia de la educación en la Península» (2003), monográfico, de Eixo Atlántico. Revista de Pensamento. En el campo de la difusión participó como coordinador y autor en la elaboración de múltiples entradas de la Enciclopedia Galega Universal, entre los anos 1999 y 2008, así como en el caso del Dicionario Galego de Pedagoxía [J. A. Caride Gómez y Trillo Alonso, F. (dirs.), Vigo: Xunta de Galicia-Editorial Galaxia, 2010]; fue co-director con Ricardo Gurriarán de la serie virtual «Album de la Ciencia» difundida a lo largo de 2007, bajo el patrocinio del Consello da Cultura Galega: una colectánea de 50 biografías de gallegos y de gallegas del campo docente e investigador, que fueron becarios de la Junta para Ampliación de Estudios e Investigaciones Científicas (www.culturagalega,org/albumdaciencia/); así como documentalista en temática histórico-educativa de dos programas de la serie de la TVG sobre historia de Galicia (2004, 2008), en tres filmes de Acuarela Comunicación: Anxel Casal. A luz impresa (2005), Crónicas de pizarra e xiz (2006), y Crónicas da represión lingüística (2008), de la muestra gráfica y audiovisual Lembranzas da infancia, recordos da escola (2011) y, con otros autores, del programa conmemorativo de la Constitución de 1812 (2012); co-editor del n. 50 de la Revista Galega de Educación (2011), en el que se hace balance de los 25 años de vida de dicha publicación editada por Nova Escola Galega, y, con Xosé M. Malheiro, del monográfico «As Escolas dos Emigrantes» en el n. ${ }^{\circ} 53$ de la mencionada revista en 2012, habiendo merecido en 2005 varios premios por la Historia da educación e da cultura en Galicia (Séculos IV-XX). Permanencias e cambios no contexto cultural e educativo europeo (Vigo: Xerais, 2004). 
También Narciso de Gabriel Fernández mereció diversos premios en 2002 por el libro Escolantes e escolas de ferrado (Vigo: Xerais, 2001), así como por la investigación Ler e escribir en Galicia. A alfabetización dos galegos e as galegas nos séculos XIX e XX , editada luego en 2006. Fue comisario de la Exposición " 25 anos da Universidade da Coruña».

Por su parte, Xosé Manuel Malheiro Gutiérrez mereció el Premio de investigación, modalidad tesis doctorales en la especialidad de Humanidades y Ciencias Sociales (2004), concedido por la Deputación de Pontevedra, siendo también co-editor con Alexandra Cabana, del monográfico "A Historia da Educación na Galiza», n. ${ }^{\circ}$ 27-28 de Murguía. Revista Galega de Historia (2013). Uxio Otero Urtaza fue, por su parte, comisario de la exposición «Las Misiones Pedagógicas 1931-1936», promovida en 2007 por la Fundación Francisco Giner de los Ríos, la Residencia de Estudiantes y la Sociedad Estatal de Conmemoraciones Culturales, y editor también de su catálogo. Ha dirigido, además, como se dijo, las Xornadas de Historia de la Educación de Foz.

Vicente Peña Saavedra, en calidad de coordinador científico del Museo Pedagóxico de Galicia (MUPEGA) entre los años 2000 y 2005, y en colaboración con el cuadro de profesores adscritos al Museo (entre ellos, Emilio Castro Fustes) promovió diferentes iniciativas: de organización de congresos y jornadas ya referidas; expositivas: Microespazo Mupega (2003-2006), Mesoespazo Mupega (2003-2005), O legado educativo dos galegos de Cuba (2005), Espazo Mupega (2006), Más de cen anos de prensa escolar en Galicia (2006), Ronsel de ilusións (2006), Nós mesmos: asociacionismo galego en la emigración (2008); o también promovidas por el Consello da Cultura Galega: Escolas de Habaneiros. Memoria gráfica (2008), Luces de Alén Mar. As Escolas de Americanos en Galicia (2012), y publicísticas (en soporte DVD, de A escola na retina: memoria gráfica da educación en Galicia (2003); O ensino, unha viaxe no tempo (2004) y otros quince reportajes documentales, bajo el título Educadores gallegos. Historias de vida profesional (entre 1999 y 2006); siendo, asimismo, iniciador y cogestor para los países del entorno ibérico de la Red Iberoamericana para la Investigación y Difusión del Patrimonio Histórico-Educativo (RIDPHE), y director del proyecto de investigación y aplicación multimedia As escolas da emigración, realizado en el marco del Arquivo da Emigración-Consello da Cultura Gallega. 


\section{PARA UNA EVALUACIÓN}

\section{Relevancia de las líneas de investigación desarrolladas}

Entre las principales líneas de investigación desarrolladas, encontramos las referidas a los estudios relativos al profesorado, en particular de escuela primaria y de centros públicos, durante el tiempo del primer tercio del siglo $\mathrm{xx}$, con alguna atención igualmente a las décadas finales del siglo XIX (status, formación, biografías, comportamiento socio-político, renovación metodológica...); el tratamiento de los aspectos sociales e ideológicos en relación con las políticas educativas estatales y con la administración escolar; los estudios de instituciones y centros escolares específicos; la acción educativa de los emigrantes gallegos a América; las cuestiones relativas a la historia pedagógica (corrientes, autores, influencias) y a la historia del currículum (materias, libros didácticos y otros recursos, metodología, innovaciones), y el patrimonio escolar material y los museos pedagógicos.

Con menos intensidad: las mujeres y la educación formal, así como la cuestión del género; la alfabetización desde el Antigo Régimen hasta el siglo xx; el desarrollo universitario en Santiago en el tiempo de la Ilustración y en el siglo xx; los aspectos relacionados con la docencia y la investigación en Historia de la Educación; la problemática suscitada por la identidad cultural y lingüística de Galicia; los libros y la cultura letrada, y la formación eclesiástica y religiosa.

Líneas de investigación todas ellas referidas temporalmente al siglo $\mathrm{xx}$, en particular, con ausencia casi general de análisis sobre períodos anteriores al siglo XVII, relacionadas, aunque no estrechamente, con lo que podría ser considerado como agenda (española) de investigación pedagógica. Muy atentas al escenario gallego, aunque también en parte a los desarrollos estatales más que a los internacionales.

Líneas de investigación que se han traducido en un notable número de unidades bibliográficas publicadas de modo diversificado (libros, capítulos de libros, capítulos de libros de actas congresuales, artículos de revistas), que en mayor medida plasman resultados de investigación, más que ejercicios de difusión, sin duda también presentes. Debe anotarse la alta presencia de la lengua gallega como medio de comunicación: un $60 \%$ en el caso de los libros, un $57 \%$ en el caso de artículos de revistas 
(con apreciable porcentaje de indexación, aunque con muy bajo traslado internacional) y un $43 \%$ en el caso de los capítulos de libros.

En cuanto a la relevancia, es necesario decir que la actividad investigadora realizada ha contribuido a formar y a fortalecer un cuadro de especialistas historiadores de la educación en Galicia, permitiendo además sostener el preciso diálogo epistemológico e historiográfico español e internacional. Un cuadro que se "representa» ante las comunidades científicas a través, en particular, de la publicación Sarmiento. Anuario Galego de Historia da Educación y de algunas notables ediciones que contribuyen a la difusión del conocimiento histórico. Sin embargo, es necesario señalar, como limitación y dificultad mayor, la escasez de investigaciones abordadas como tesis doctorales, un medio de alta cualificación académica e investigadora.

El conocimiento histórico aportado provee de información precisa, que contribuye a proporcionarle a los diversos sectores de la sociedad gallega una visión histórica objetiva, y frecuentemente crítica, de los fenómenos educativos (historia del presente) en diferentes aspectos. Entre ellos, la génesis y desarrollo de variadas instituciones educativas, tanto de carácter público como privado o social, los procesos de alfabetización, la apertura cultural de nuestra sociedad, las influencias recibidas, o el papel y la historia del profesorado, donde enraízan por ejemplo experiencias actuales como la del movimiento de renovación pedagógica Nova Escola Galega. Otros temas de singular relevancia son el papel jugado por las instituciones universitarias o el conflicto cultural y lingüístico que viven la sociedad gallega, las familias y las personas en el plano intelectual y en el emocional, presente en los centros de formación.

El conocimiento histórico educativo quizá no aporte información técnica para contribuir — desde el punto de la realización de tareas y de la inmediatez pragmática- a la mejora de las prácticas educativas, pero es indudable su contribución en la mejor elección de propuestas metodológicas y de política educativa.

\section{Puntos fuertes y débiles}

Entre las fortalezas, debemos señalar en primer lugar el propio conocimiento adquirido, variado y matizado; en segundo lugar, la presen- 
cia social acumulada desde el punto de vista de su difusión; por otro lado, un cierto reconocimiento académico y social alcanzado por el aludido "grupo gallego»; además, alguna disposición de mediaciones como la existencia de un Museo Pedagógico o la sensibilidad de acogida del mundo editorial gallego; por último, una cierta conciencia de grupo de investigación/intervención, que se manifesta como sentido de grupo con conciencia "galleguista» y declarada aspiración de unas buenas prácticas de representación gallega.

Sin embargo, los puntos débiles y amenazas son notables: algunos tienen que ver con el contexto académico, social y cultural, en el que goza de infeliz fuerza el presentismo pragmatista, que se traslada a las estructuras universitarias y a sus planes de estudios en forma de negación de la importancia del conocimiento histórico. Lo que se traduce en la reducción de encargos docentes, las dificultades en la formalización de grupos de investigación en este ámbito, y la desconsideración de lo que podrían ser "políticas de excepción cultural». ${ }^{31} \mathrm{El}$ propio contexto social y político de Galicia, con su extraordinario debilitamiento demográfico, entre otras carencias, retira vigor biológico y social al proyecto de Galicia como sujeto histórico y político, lo que, a su vez, condiciona la reducción de investigadores e investigadoras dispuestos a afrontar su horizonte investigador en el campo de la historia de la educación.

Otros puntos débiles tienen más que ver con el estatuto de los actuales investigadores e investigadoras. Empieza a observarse un cierto declive del que llamamos "grupo gallego», en si mismo reducido en número (con sus ciclos de desempeño profesional avanzados). De este modo, incluso hablando de un consistente grupo de investigadores, éstos no consiguen dirigir un suficiente grupo de tesis doctorales en este campo, lo que difi-

\footnotetext{
${ }^{31}$ Entiéndase como el sentido político cultural que reconoce la importancia de favorecer, desde los intereses colectivos, unos determinados horizontes. Sin ese sentido, en la actualidad hay campos de las ciencias sociales y de las humanidades en Galicia que difícilmente pueden dar los frutos que potencialmente podrían dar, en tanto se ven sometidos a dinámicas de control académico contradictorias y exteriores con la observación de Galicia como comunidad democrática y sujeto histórico. Hablamos, por ejemplo, de la influencia de la aplicación exterior de criterios de valoración de la productividad científica, que deben existir sin duda, desde el cuadro de criterios diseñado por el Social Sciences Citation Index. A este respecto queremos hacer notar la diferencia existente entre lo que podríamos llamar el espacio científico gallego de ciencias sociales y de humanidades o el catalán, abundantemente más protegido éste último, tanto por su más densa malla universitaria como por la presencia firme del Institut d'Estudis Cataláns, dotado de oportunos recursos y con capacidad de edición de una notable serie de publicaciones académicas.
} 
culta su renovación. Por otro lado, las actividades de investigación que se llevan a cabo deberían obedecer actualmente, en alguna medida, a una agenda y programa de investigación sistemático reconocibles, más allá de las preocupaciones individuales y de la creatividad intelectual de los investigadores; en el conjunto producido se puede observar una insuficiente reflexividad y reconocimiento crítico de lo que son los debates epistemológicos e historiográficos, con consecuencias en expresiones un algo frecuentes de eclecticismo y de imprecisión teórica, que penalizan la calidad y el valor de los productos de la investigación realizada. ${ }^{32}$ Es necesario señalar, por fin, su insuficiente interrelación académica internacional en el campo de la historia de la educación.

\section{Investigadores en contexto}

Estamos ante una producción de cerca de 500 unidades bibliográficas elaboradas por un total de 110 autores (un 35\% de ellas autoras), debiendo señalarse que en un $75 \%$ de los casos se trata de intervenciones esporádicas (casi siempre con una única contribución en todo el período considerado de 13 años). Por eso, deberíamos hablar de un menor número de autores y autoras con distinto grado de atención hacia la historia de la educación: unos 25, de ellos siete mujeres. Dentro de este conjunto es donde debemos situar a los 10 que tienen o han tenido en el período una producción más regular y continuada, tal como hemos tenido ocasión de ver en los varios apartados analíticos anteriores: 318 unidades bibliográficas de las 499 censadas; es decir, algo más del 65\% del total. De estos 25, la gran mayoría son doctores universitarios docentes en este ámbito, aunque también hay algunos en el ámbito de la educación secundaria o en centros de formación teológica, una especialista en biblioteconomía y un periodista de larga trayectoria.

En conjunto expresan el pluralismo de la investigación presente en las llamadas «sociedades abiertas», y por ello son también variadas las razones y factores que mueven sus empeños de investigación y de difusión

\footnotetext{
${ }^{32}$ Los autores que inspiran el desarrollo historiográfico de nuestro tiempo, entre los que podríamos señalar a Burke, Chartier, Certeau, Darnton, Elias, Foucault, Bourdieu, Escolano, Viñao, Thompson, R. Williams, Julià, Goodson, Ginzbourg, o Nóvoa, están limitadamente presentes en la constitución de las tramas teóricas y conceptuales que asientan las bases intelectuales utilizadas por los investigadores gallegos.
} 
del conocimiento alcanzado; del mismo modo, son diferentes los factores condicionantes que inciden en los asuntos que estudian, en los silencios que deciden, o en los discursos que emiten, siendo también distintas, en orden de prioridades, las audiencias a quien dirigen su comunicación, y, finalmente distinta la conciencia histórica que tienen de sí mismos.

\section{Nuevas líneas a desarrollar}

Expuesto con alguna brevedad, diremos que podrá ser oportuno: intensificar los estudios sobre historia curricular y sobre la construcción social y profesional del currículum (debate de programas de materias, elaboración de libros de texto y de recursos didácticos, los mercados,...), en particular desde los pasados años 70; examinar los tiempos de la Transición democrática desde los finales del Franquismo; profundizar todo el espacio diverso de la educación social y de la educación popular; estudiar el papel de la Iglesia católica en los procesos educativos y en la conformación de la ciudadanía; abrir los estudios sobre la historia de la infancia y de la educación infantil, así como de la educación profesional, hasta el presente sólo esbozados; observar las diversas dinámicas sociales no formales y el papel de los actores sociales; generar una observación histórica, genealógica y crítica, con auxilio de la sociología histórica, de la constitución de las redes y experiencias de enseñanza privada en la historia de la Galicia contemporánea; abordar el estudio de los discursos políticos (semántica y pragmática) en relación con la educación en la Galicia autonómica; examinar, desde la observación etnográfica y desde la antropología cultural, el conflicto cultural-lingüístico existente y su presencia en las instituciones escolares; abrir el examen de nuevas fuentes documentales, como las que provienen del lenguaje icónico; analizar el campo de las instituciones y de la educación que atiende necesidades educativas específicas; profundizar en la cuestión de género como factor de desigualdad social o, estudiar la conformación de las identidades sociales, culturales y cívicas, sin olvidar el estudio de la profesionalización docente en las décadas finales del siglo $\mathrm{xx}$, de las dinámicas y movilizaciones de los profesores como actores relevantes en los procesos educativos (que leen, escriben, intervienen...) y de las trayectorias de vida de los educadores y educadoras. Hay, pues, todo un amplio abanico de nuevos objetos de investigación con proximidad al presente, sin por ello olvidar otros distanciados en el tiempo, en tanto que Galicia es un pueblo de larga historia social y cultural. 
Figura 1. Distribución porcentual temática de la produccion histórico-educativa: Artículos. Periodo 2002-2014

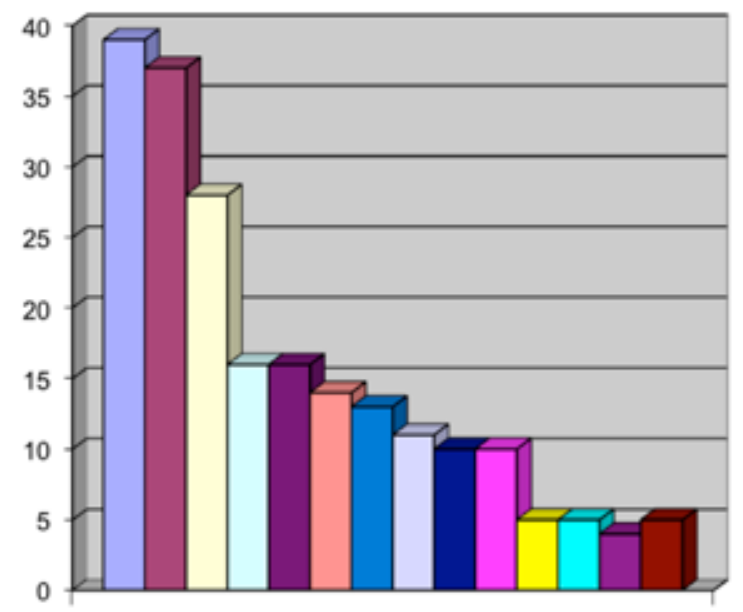

口profesorado

口. $\mathrm{H}^{\mathrm{a}}$ Pedagogia

口 Alfabetización, ed. popular y emigración

口 Historia del curriculum, metodología

a Historia de las instituciones

口patrimonio escolar

口Politica educativa

口Educación de las mujeres y alfabetización

- Identidad cultural y lingüistica

口Educación secundaria

DEstatuto de la infancia

口Docencia e historiografia educativa

口Historia de la literatura infantil

-Otros temas

Fuente: Elaboración propia.

Figura 2. Distribución temática de la producción histórico-educativa (n. de unidades): libros. Periodo 2002-2014

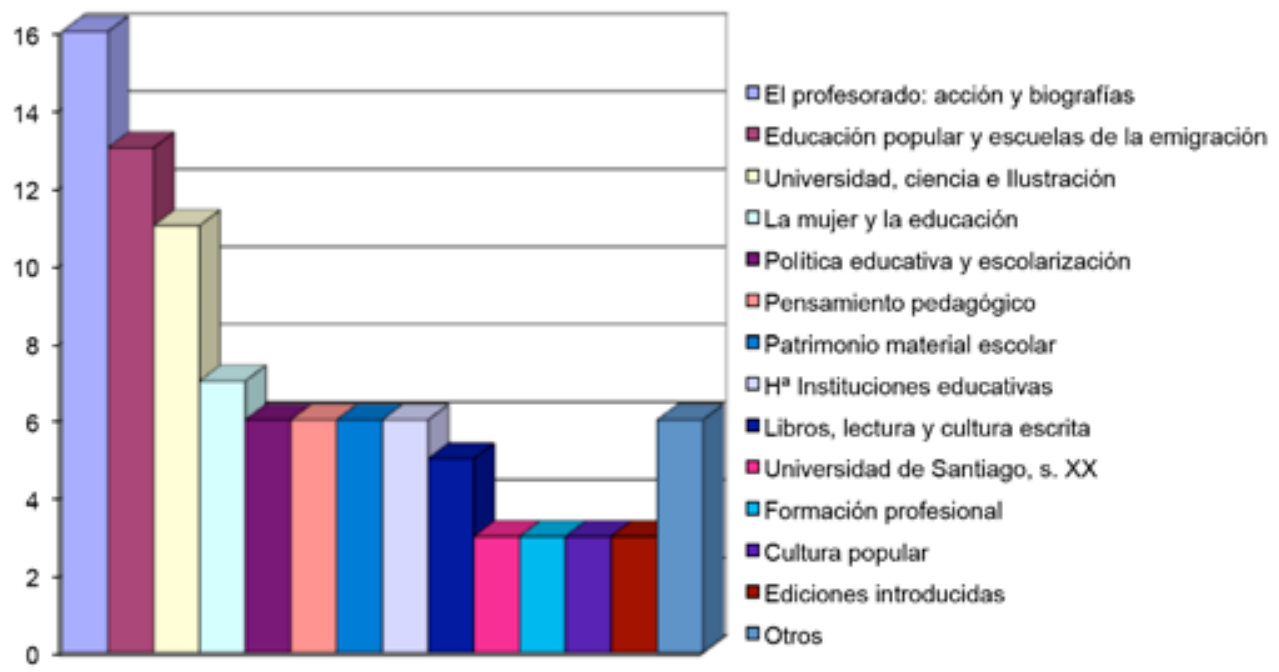

Fuente: Elaboración propia. 


\section{Nota sobre los autores:}

Antón Costa Rico es Catedrático de Historia da Educación en la Universidade de Santiago de Compostela. Con trayectoria docente e investigadora en los campos de la política educativa y de la historia da educación. Es uno de los fundadores del movimiento de renovación pedagógica Nova Escola Galega, del cual es militante activo. Ex-director del Instituto de Ciencias de la Educación de la Universidad de Santiago y ex-decano de la Facultad de Ciencias de la Educación. Fue presidente de la SEDHE. Es miembro de consejos de redacción y/o asesores científicos de varias publicaciones científicas. Entre sus obras: Escolas e mestres (1989), Informe sobre a situación e estado do sistema educativo en Galicia (1995; Dir. de Informe), Fray Martín Sarmiento. La educación de la niñez y de la juventud (en colaboración) (2002), Historia da educación e da cultura en Galicia (2004), A construción do coñecemento pedagóxico Antecedentes e desenvolvementos no século XX (2009). Entre sus trabajos de análisis historiográfico: «Los manuales de Historia de la Educación y de la Pedagogía: una tradición textual revisada» (Firenze, 2015); «Historia de la educación en España. Realidades, problemas y tendencias en el dominio de la investigación» (Uberlândia, Brasil, 2011); «A docencia da Historia da Pedagoxía/Historia da Educación en España: institucionalización, textos e rotas» (Uberlândia, Brasil, 2009); «Iluminar la intensa historia de las prácticas escolares desde la historiografía educativa: potencialidad y retos» (Rio de Janeiro, 2012).

Xosé M. Malheiro Gutiérrez. (Moxico, Angola, 1961) es Doctor en Filosofía y Ciencias de la Educación por la Universidad de Santiago de Compostela y Profesor Contratado Doctor en el Dpto. de Pedagoxía e Didáctica de la Facultad de Ciencias de la Educación de la Universidade da Coruña. Anteriormente lo fue en la de Vigo (2001-2002) y la de Santiago (20032008). Ha publicado diferentes trabajos sobre la intervención educativa de la emigración gallega a América y ha ganado, además, varios premios relacionados con la investigación histórico-educativa. Es miembro de los grupos de investigación ESCULCA (USC) y Política educativa, Historia e Sociedade (UDC). Pertenece a la SEDHE y al consejo de redacción de su revista científica y órgano de expresión de la Sociedad, Historia y Memoria de la Educación. Es miembro del movimiento de renovación pedagógica Nova Escola Galega. Investiga sobre los procesos migratorios y sus implicaciones en la escuela; el contenido de los manuales escolares; y el empleo de la fotografía y el cine como recursos para el estudio de la memoria escolar. 